\title{
Radion stabilization in higher curvature warped spacetime
}

\author{
Ashmita Das $^{1, \mathrm{a}}$, Hiya Mukherjee ${ }^{2, \mathrm{~b}}$, Tanmoy Paul ${ }^{2, \mathrm{c}}$, Soumitra SenGupta ${ }^{2, \mathrm{~d}}$ \\ ${ }^{1}$ Department of Physics, Indian Institute of Technology, North Guwahati, Guwahati, Assam 781039, India \\ 2 Department of Theoretical Physics, Indian Association for the Cultivation of Science, 2A \& 2B Raja S.C. Mullick Road, Kolkata 700 032, India
}

Received: 23 December 2017 / Accepted: 31 January 2018 / Published online: 6 February 2018

(C) The Author(s) 2018. This article is an open access publication

\begin{abstract}
We consider a five dimensional AdS spacetime in presence of higher curvature term like $F(R)=R+\alpha R^{2}$ in the bulk. In this model, we examine the possibility of modulus stabilization from the scalar degrees of freedom of higher curvature gravity free of ghosts. Our result reveals that the model stabilizes itself and the mechanism of modulus stabilization can be argued from a geometric point of view. We determine the region of the parametric space for which the modulus (or radion) can to be stabilized. We also show how the mass and coupling parameters of radion field are modified due to higher curvature term leading to modifications of its phenomenological implications on the visible 3-brane.
\end{abstract}

\section{Introduction}

Till date, Standard Model (SM) of particle Physics is a widely accepted theory to describe the interactions of fundamental particles. Despite its enormous successes, the model is plagued with divergence of the Higgs mass due to radiative corrections which may run up to Planck scale. An unnatural fine tuning is needed to confine the Higgs mass within $\mathrm{TeV}$ scale.

Many attempts have been made to address this problem by considering the theories beyond SM of particle Physics. Few such candidates are - supersymmetry, technicolor and extra dimensions. Among many such attempts [1-15], RandallSundrum (RS) model [6] of warped extra dimension draws special attention since it resolves the gauge hierarchy problem without choosing any intermediate scale in the theory.

In RS model, two 3-branes are embedded in a five dimensional anti de-Sitter spacetime compactified on a $M_{4} \times S_{1} / Z_{2}$ orbifold. The distance between these two branes is assumed

\footnotetext{
a e-mail: ashmita@iitg.ernet.in

b e-mail: tphm@iacs.res.in

c e-mail: tpap@iacs.res.in

d e-mail: tpssg@iacs.res.in
}

to be $\sim$ of Planck length so that the required hierarchy between the two branes is generated. One of the crucial aspects of this braneworld scenario is to stabilize the distance between the branes (known as modulus or radion). For this purpose, it is necessary to generate an appropriate radion potential with a stable minimum consistent with the value proposed in RS model in order to solve the hierarchy problem. Goldberger and Wise (GW) proposed a mechanism [16] to create such a radion potential by introducing a bulk scalar field with appropriate boundary values. Subsequently the phenomenology of radion field has also been studied extensively. This radion phenomenology [17-20] along with the study of RS graviton [21-25] and RS black holes [26$28]$ are considered to be the testing ground of warped extra dimensional models in collider experiments $[29,30]$. As the present experimental lower bound of the first graviton KKmode mass climbs above $3 \mathrm{TeV}$, the RS-type resolution of the naturalness problem is undoubtedly under pressure. The question that how the Higgs is so much lighter than the 5dimensional Planck scale needs to be settled properly. However, in any higher dimensional model with gravity in the bulk, the modulus must be stabilized to appropriate value to extract a meaningful low energy effective theory on the brane. The present work aims to address this issue specially in the context of a higher dimensional model where the fundamental curvature scale is of the order of Planck scale.

It is well known that Einstein-Hilbert action can be generalized by adding higher order curvature terms which naturally arise from diffeomorphism property of the action. Such terms also have their origin in String theory due to quantum corrections. $F(R)$ [31-33], Gauss-Bonnet (GB) [34-36] or more generally Lanczos-Lovelock gravity are some of the candidates in higher curvature gravitational theory.

Higher curvature terms become extremely relevant at the regime of large curvature. Thus for RS bulk geometry where the curvature is of the order of Planck scale, the higher curvature terms should play a crucial role. In general inclusion of higher curvature terms in the action leads to the appearance of 
ghost from higher derivative terms resulting into Ostragradsky instability. The Gauss-Bonnet model (a special case of Lanczos-Lovelock model) is however free of this instability due to appropriate choice of various quadratic combinations of Riemann tensor, Ricci tensor and curvature scalar. Some important modified solutions of the Randall-Sundrum model in presence of Gauss-Bonnet terms have been obtained by Kim et al. [37,38] in the context of both static and inflationary scenario. A GB modified warped solution and it's phenomenological implications was also discussed in [39].

In contrast to GB model $F(R)$ gravity model however contains higher curvature terms consisting only of the scalar curvature $R$. Once again just as GB model, certain classes of $F(R)$ gravity models are free from ghost-like instability (See Sect. 3 for a detailed discussion). In general $F(R)$ model can be mapped into a scalar-tensor theory at the action level by a conformal transformation of the metric $[31,32,40-$ 45]. The issue of instability of the original $F(R)$ model is now reflected in the form of the kinetic and potential terms of the scalar field in the dual scalar-tensor model, where the potential will have a stable minimum and a kinetic term with proper signature if the original $F(R)$ model is free from ghosts (see Sect. 3). It is know that to achieve modulus stabilization in RS-like model, one needs a scalar field [16]. We, in this work, therefore look for the possibility of having a geometric modulus stabilization mechanism due to the scalar degree of freedom originating from the higher curvature terms. The important questions in this context are:

- Is the RS braneworld modified by $F(R)$ gravity, stabilized even without introducing an external stabilizing field?

- If the modulus can be stabilized in the dual scalar-tensor model, does it mean that it is also stabilized in the original $F(R)$ model?

- Does the scalar kinetic and potential terms for the purpose of modulus stabilization correspond to a $F(R)$ model which is free of ghost-like instability?

- If the braneworld scenario is stabilized consistently then how the radion mass and coupling parameters will change from that of RS scenario due to the presence of higher order curvature terms?

We aim to address these questions in this work by using the equivalence between $F(R)$ and scalar-tensor theory.

The paper is organized as follows: Following two sections are devoted to brief reviews of RS scenario and conformal relationship between $F(R)$ and scalar-tensor (ST) theory. In Sect. 4, we extend our analysis of Sect. 3 for the specific $F(R)$ model considered in this work. Section 5 extensively describes the modulus stabilization, radion mass and coupling for the dual ST model while Sect. 6 addresses these for the original $F(R)$ model. After discussing the equivalence, the paper ends with some conclusive remarks in Sect. 7.

\section{Brief description of RS scenario and its stabilization via GW mechanism}

RS scenario is defined on a five dimensional AdS spacetime involving one warped and compact extra spacelike dimension. Two 3-branes known as TeV/visible and Planck/hidden brane are embedded in five dimensional spacetime where the intermediate region between the branes is termed as 'bulk'. If $\phi$ is the extra dimensional angular coordinate, then the branes are located at two fixed points $\phi=(0, \pi)$ while the latter one is identified with our known four dimensional universe. The opposite brane tensions along with the finely tuned five dimensional cosmological constant serve as energymomentum tensor of RS scenario. The resulting spacetime metric [6] is non-factorizable and expressed as,

$d s^{2}=e^{-2 k r_{c}|\phi|} \eta_{\mu \nu} d x^{\mu} d x^{\nu}-r_{c}^{2} d \phi^{2}$

Here, $r_{c}$ is the compactification radius of the extra dimension. Due to $S^{1} / Z_{2}$ compactification along the extra dimension, $\phi$ ranges from $-\pi$ to $+\pi$. The quantity $k=\sqrt{\frac{-\Lambda}{12 M^{3}}}$, is of the order of 5-dimensional Planck scale $M$. Thus $k$ relates the 5D Planck scale $M$ to the 5D cosmological constant $\Lambda$.

All the dimensionful parameters described above are related to the reduced 4-dimensional Planck scale $M_{P l}$ as,

$M_{P l}^{2}=\frac{M^{3}}{k}\left(1-e^{-2 k \pi r_{c}}\right)$

In order to solve the hierarchy problem, it is assumed in RS scenario that the branes are separated by such a distance that $k \pi r_{c} \approx 36$. Then the exponential factor present in the metric, which is often called warp factor, produces a large suppression so that a mass scale of the order of Planck scale is reduced to $\mathrm{TeV}$ scale on the visible brane. A scalar mass say mass of Higgs is given as,

$m_{H}=m_{0} e^{-k \pi r_{c}}$

where $m_{H}$ and $m_{0}$ are physical and bare Higgs mass respectively. But one of the crucial aspects of this braneworld scenario is to stabilize the distance between the branes (known as modulus or radion). For this purpose, Goldberger and Wise demonstrated that the modulus corresponding to the radius of the extra dimension in RS warped geometry model can be stabilized [16] by invoking a massive scalar field in the bulk with appropriate vacuum expectation values (vev) at the two 3-branes that reside at the orbifold fixed points. Consequently the phenomenology of the radion field originating from 5D gravitational degrees of freedom has also been explored [17]. 


\section{Transformation of a $F(R)$ theory to scalar-tensor theory}

In this section, we briefly describe how a higher curvature $\mathrm{F}(\mathrm{R})$ gravity model in five dimensional scenario can be recast into Einstein gravity with a scalar field. The $F(R)$ action is expressed as,

$S=-\frac{1}{2 \kappa^{2}} \int d^{4} x d \phi \sqrt{G} F(R)$

where $x^{\mu}=\left(x^{0}, x^{1}, x^{2}, x^{3}\right)$ are usual four dimensional coordinate and $\phi$ is the extra dimensional spatial angular coordinate. $R$ is the five dimensional Ricci curvature and $G$ is the determinant of the metric. Moreover $\frac{1}{2 \kappa^{2}}$ as taken as $2 M^{3}$ where $M$ is the five dimensional Planck scale. Introducing an auxiliary field $A(x, \phi)$, above action (4) can be equivalently written as,

$S=-\frac{1}{2 \kappa^{2}} \int d^{4} x d \phi \sqrt{G}\left[F^{\prime}(A)(R-A)+F(A)\right]$

By the variation of the auxiliary field $A(x, \phi)$, one easily obtains $A=R$. Plugging back this solution $A=R$ into action (5), initial action (4) can be reproduced. At this stage, perform a conformal transformation of the metric as

$G_{M N}(x, \phi) \rightarrow \tilde{G}_{M N}=\exp \left(\sigma(x, \phi) G_{M N}(x, \phi)\right.$

$M, N$ run form 0 to $5 . \sigma(x, \phi)$ is conformal factor and related to the auxiliary field as $\sigma=(2 / 3) \ln F^{\prime}(A)$. If $R$ and $\tilde{R}$ are the Ricci scalar formed by $G_{M N}$ and $\tilde{G}_{M N}$ respectively, then they are related as,

$R=e^{\sigma}\left[\tilde{R}-4 \tilde{G}^{M N} \partial_{M} \partial_{N} \sigma-3 \tilde{G}^{M N} \partial_{M} \sigma \partial_{N} \sigma\right]$

Due to the above relation between $R$ and $\tilde{R}$, action (5) turns out to be,

$$
\begin{aligned}
S= & \int d^{4} x d \phi \sqrt{\tilde{G}}\left[-\frac{1}{2 \kappa^{2}} e^{-\frac{3 \sigma}{2}} F^{\prime}(A)\left(\tilde{R}-4 \tilde{G}^{M N} \partial_{M} \partial_{N} \sigma\right.\right. \\
& \left.\left.-3 \tilde{G}^{M N} \partial_{M} \sigma \partial_{N} \sigma\right)-\frac{1}{2 \kappa^{2}} e^{-\frac{5 \sigma}{2}}\left(F(A)-A F^{\prime}(A)\right)\right]
\end{aligned}
$$

Considering $F^{\prime}(R)>0$ and using the aforementioned relation between $\sigma$ and $F^{\prime}(A)$, one lands up to the following scalar-tensor action

$$
\begin{aligned}
S= & \left(1 / 2 \kappa^{2}\right) \int d^{4} x d \phi \sqrt{\tilde{G}}\left[-\tilde{R}+3 \tilde{G}^{M N} \partial_{M} \sigma \partial_{N} \sigma\right. \\
& \left.-\frac{1}{2 \kappa^{2}}\left(\frac{F(A)}{F^{\prime}(A)^{5 / 3}}-\frac{A}{F^{\prime}(A)^{2 / 3}}\right)\right]
\end{aligned}
$$

where $\tilde{R}$ is the Ricci scalar formed by $\tilde{G}_{M N} \cdot \sigma(x, \phi)$ is the scalar field, emerging from higher curvature degrees of freedom. At this stage, it is important to note that for $F^{\prime}(R)<0$, the kinetic term of the scalar field $\sigma$ as well as the Ricci scalar $\tilde{R}$ in the above action come with wrong sign, which indicates the existence of ghost field. Thus to avoid the ghost like structure, $F^{\prime}(R)$ must be greater than zero. Later we shall show that in the context of the present work, this condition is indeed satisfied.

The kinetic part of $\sigma(x, \phi)$ in Eq. (30), though correct in sign, is non-canonical. In order to make the scalar field canonical, transform $\sigma \rightarrow \Phi(x, \phi)=\sqrt{3} \frac{\sigma(x, \phi)}{\kappa}$. In terms of $\Phi(x, \phi)$, the above action takes the form,

$S=\int d^{4} x d \phi \sqrt{\tilde{G}}\left[-\frac{\tilde{R}}{2 \kappa^{2}}+\frac{1}{2} \tilde{G}^{M N} \partial_{M} \Phi \partial_{N} \Phi-V(\Phi)\right]$

where $V(\Phi)=\frac{1}{2 \kappa^{2}}\left[\frac{F(A)}{F^{\prime}(A)^{5 / 3}}-\frac{A}{F^{\prime}(A)^{2 / 3}}\right]$ is the scalar field potential which depends on the form of $F(R)$. Thus the action of $F(R)$ gravity in five dimension can be transformed into the action of a scalar-tensor theory by a conformal transformation of the metric.

\section{RS like spacetime in $F(R)$ model and corresponding scalar-tensor theory}

In the present work, we consider a five dimensional AdS spacetime with two 3-brane scenario in $F(R)$ model. To the leading order in higher curvature term, the form of $F(R)$ is taken as $F(R)=R+\alpha R^{2}$ where $\alpha$ is a constant with square of the inverse mass dimension. Considering $\phi$ as the extra dimensional angular coordinate, two branes are located at $\phi=0$ (hidden brane) and at $\phi=\pi$ (visible brane) respectively while the latter one is identified with the visible universe. Moreover the extra dimension is $S^{1} / Z_{2}$ orbifolded along the coordinate $\phi$. The action for this model is:

$$
\begin{aligned}
S= & \int d^{4} x d \phi \sqrt{G}\left[-\frac{1}{2 \kappa^{2}}\left(R+\alpha R^{2}\right)-\Lambda\right. \\
& \left.-V_{h} \delta(\phi)-V_{v} \delta(\phi-\pi)\right]
\end{aligned}
$$

where $\Lambda(<0)$ is the bulk cosmological constant and $V_{h}, V_{v}$ are the brane tensions on hidden, visible brane respectively.

This higher curvature like $F(R)$ model (in Eq. (8)) can be transformed into scalar-tensor theory by using the technique discussed in the previous section. Performing a conformal transformation of the metric as

$$
G_{M N}(x, \phi) \rightarrow \tilde{G}_{M N}=\exp \left(\frac{1}{\sqrt{3}} \kappa \Phi(x, \phi)\right) G_{M N}(x, \phi)
$$

the above action (in Eq. (8)) can be expressed as a scalartensor theory with the action given by:

$$
S=\int d^{4} x d \phi \sqrt{\tilde{G}}\left[-\frac{\tilde{R}}{2 \kappa^{2}}+\frac{1}{2} \tilde{G}^{M N} \partial_{M} \Phi \partial_{N} \Phi-V(\Phi)\right.
$$




$$
\begin{aligned}
& -\Lambda-\exp \left(-\frac{5}{2 \sqrt{3}} \kappa \Phi\right) V_{h} \delta(\phi) \\
& \left.-\exp \left(-\frac{5}{2 \sqrt{3}} \kappa \Phi\right) V_{v} \delta(\phi-\pi)\right]
\end{aligned}
$$

where the quantities in tilde are reserved for ST theory. $\tilde{R}$ is the Ricci curvature formed by the transformed metric $\tilde{G}_{M N}$. $\Phi(x, \phi)$ is the scalar field corresponds to higher curvature degrees of freedom and $V(\Phi)$ is the scalar potential which for this specific choice form of $F(R)$ has the form,

$$
\begin{aligned}
V(\Phi)= & -\frac{1}{8 \kappa^{2} \alpha} \exp \left(-\frac{5}{2 \sqrt{3}} \kappa \Phi\right)\left[\exp \left(\frac{3}{2 \sqrt{3}} \kappa \Phi\right)-1\right]^{2} \\
& +\Lambda\left[\exp \left(-\frac{5}{2 \sqrt{3}} \kappa \Phi\right)-1\right]
\end{aligned}
$$

One can check that the above potential (in Eq. (11)) is stable for the parametric regime $\alpha<0$. This immediately ensures that for our model with negative bulk curvature, $F^{\prime}(R)=1+2 \alpha R$ is always greater than zero which in turn indicates that the original $F(R)$ model is free from Ostragradsky instability as discussed earlier.

The stable value $(<\Phi>)$ as well as the mass squared $\left(m_{\Phi}^{2}\right)$ of the scalar field $(\Phi)$ are given by the following two equations

$\exp \left(\frac{3}{2 \sqrt{3}} \kappa<\Phi>\right)=\left[\sqrt{9-40 \kappa^{2} \alpha \Lambda}-2\right]$

and

$m_{\Phi}^{2}=-\frac{1}{8 \alpha}\left[\sqrt{9-40 \kappa^{2} \alpha \Lambda}\right]\left[\sqrt{9-40 \kappa^{2} \alpha \Lambda}-2\right]^{-\frac{2}{3}}$

The above two equations imply that in order to have consistent values for $<\Phi>$ and $m_{\Phi}^{2}, \alpha$ and $\Lambda$ must satisfy the condition $\kappa^{2} \alpha \Lambda<\frac{1}{8}$.

Furthermore, the minimum value of the potential i.e. $V(<$ $\Phi>$ ) is non zero and serves as a cosmological constant. Thus the effective cosmological constant in scalar-tensor theory is $\Lambda_{e f f}=\Lambda+V(<\Phi>)$ where $V(<\Phi>)$ is,

$$
\begin{aligned}
V(<\Phi>)= & -\Lambda+\left[\sqrt{9-40 \kappa^{2} \alpha \Lambda}-2\right]^{-\frac{5}{3}} \\
& {\left[\Lambda-\left(1 / 8 \kappa^{2} \alpha\right)\left[\sqrt{9-40 \kappa^{2} \alpha \Lambda}-3\right]^{2}\right] }
\end{aligned}
$$

Above form of $V(<\Phi>)$ with $\Lambda<0$ clearly indicates that $\Lambda_{\text {eff }}$ is also negative or more explicitly,the corresponding scalar-tensor theory for the original $F(R)$ model has an AdS like spacetime. Considering $\xi$ as the fluctuation of the scalar field over its vev, the final form of action for the scalar-tensor theory in the bulk can be written as,

$$
\begin{aligned}
S= & \int d^{4} x d \phi \sqrt{\tilde{G}}\left[-\frac{\tilde{R}}{2 \kappa^{2}}+\frac{1}{2} \tilde{G}^{M N} \partial_{M} \xi \partial_{N} \xi\right. \\
& \left.-(1 / 2) m_{\Phi}^{2} \xi^{2}-\Lambda_{e f f}\right]
\end{aligned}
$$

where the terms up to quadratic order in $\xi$ are retained for $\kappa \xi<1$. A detailed justification of neglecting the higher order terms as well as their possible effects will be discussed in Sect. 6.3.

\section{Modulus stabilization, radion mass and coupling in scalar-tensor (ST) theory}

\subsection{Modulus stabilization}

In order to stabilize the modulus in ST theory, here we adopt the GW mechanism [16] which requires a massive scalar field in the bulk. For the case of ST theory presented in Eq. (15), $\xi$ can act as a bulk scalar field with the mass given by Eq. (13). Considering a negligible backreaction of the scalar field $(\xi)$ on the background spacetime, the solution of metric $\tilde{G}_{M N}$ is exactly same as RS model i.e.

$d \tilde{s}^{2}=e^{-2 k r_{c}|\phi|} \eta_{\mu \nu} d x^{\mu} d x^{\nu}-r_{c}^{2} d \phi^{2}$

where $k=\sqrt{\frac{-\Lambda_{e f f}}{24 M^{3}}}$. With this metric, the scalar field equation of motion in the bulk is following,

$$
\begin{aligned}
& -\frac{1}{r_{c}^{2}} \partial_{\phi}\left[\exp \left(-4 k r_{c}|\phi|\right) \partial_{\phi} \xi\right] \\
& +m_{\Phi}^{2} \exp \left(-4 k r_{c}|\phi|\right) \xi(\phi)=0
\end{aligned}
$$

where the scalar field $\xi$ is taken as function of extra dimensional coordinate only. Considering non zero value of $\xi$ on branes, the above Eq. (17) has the general solution,

$\xi(\phi)=e^{2 k r_{c}|\phi|}\left[A e^{v k r_{c}|\phi|}+B e^{-v k r_{c}|\phi|}\right]$

with $v=\sqrt{4+m_{\Phi}^{2} / k^{2}}$. Moreover $A$ and $B$ are obtained from the boundary conditions, $\xi(0)=v_{h}$ and $\xi(\pi)=v_{v}$ as follows:

$A=\frac{1}{\left(1-e^{-2 v k \pi r_{c}}\right)}\left[v_{v} e^{-(2+v) k r_{c} \pi}-v_{h} e^{-2 v k r_{c} \pi}\right]$

and

$B=\frac{1}{\left(1-e^{\left.-2 v k \pi r_{c}\right)}\right.}\left[v_{h}-v_{v} e^{-(2+v) k r_{c} \pi}\right]$

Plugging back the solution of $\xi(\phi)$ (Eq. (18)) into scalar field action and integrating over $\phi$ yields an effective modulus potential having the following form,

$$
\begin{aligned}
& V_{e f f}\left(r_{c}\right)=k(v+2) A^{2}\left(e^{2 v k r_{c} \pi}-1\right) \\
& +k(v-2) B^{2}\left(1-e^{-2 v k r_{c} \pi}\right)
\end{aligned}
$$

where $A$ and $B$ are given in Eqs. (19) and (20) respectively. This potential has a minimum at

$k \pi r_{c}=\frac{4 k^{2}}{m_{\Phi}^{2}} \ln \left[\frac{v_{h}}{v_{v}}\right]$ 
According to GW mechanism [16], the branes are stabilized at that separation for which the modulus potential becomes minimum. Thus the above equation represents the stabilized value for inter-brane separation. Expression of $m_{\Phi}^{2}$ (Eq. (13)) clearly indicates that $r_{c}$ in Eq. (22) is positive only for $\alpha<0$. Thus the $F(R)$ model depicted in Eq. (8) with positive $\alpha$ can be transformed to a scalar-tensor theory where the scalar field has negative squared mass and the modulus of the ST theory can not be stabilized.

At this stage, we mention that the value of the stabilized modulus should be $k r_{c} \simeq 12$, in order to solve the gauge hierarchy problem. Equation (22) clearly indicates that such magnitude of $k r_{c}$ can be achieved without any fine tuning of the parameters [16]. For example, $v_{h} / v_{v}=1.5$ and $m_{\Phi} / k=$ 0.2 yields $k r_{c} \simeq 12$ [16].

It may be observed that the scalar field degrees of freedom is related to the curvature as,

$\xi(\phi)=\frac{2}{\sqrt{3} \kappa} \ln [1+2 \alpha R]-<\Phi>$

Recall that $\left\langle\Phi>=\frac{2}{\sqrt{3} \kappa} \ln \left[\sqrt{9-40 \kappa^{2} \alpha \Lambda}-2\right]\right.$.

From the above expression, we can relate the boundary values of the scalar field (i.e $\xi(0)=v_{h}$ and $\xi(\pi)=v_{v}$ ) with the Ricci scalar as,

$v_{h}=\frac{2}{\sqrt{3} \kappa} \ln \left[\frac{1+2 \alpha R(0)}{\sqrt{9-40 \kappa^{2} \alpha \Lambda}-2}\right]$

and

$v_{v}=\frac{2}{\sqrt{3} \kappa} \ln \left[\frac{1+2 \alpha R(\pi)}{\sqrt{9-40 \kappa^{2} \alpha \Lambda}-2}\right]$

where $R(0)$ and $R(\pi)$ are the values of the curvature on Planck and TeV brane respectively. In Sect. 6.1, we derive the expression of the bulk scalar curvature which in this scenario becomes dependent on the bulk coordinate $y$. Thus the parameters that are used in the scalar-tensor theory are actually related to the parameters of the original $F(R)$ theory.

Furthermore to derive the stabilization condition in scalartensor theory, the backreaction of the scalar field on spacetime geometry is neglected. It can be shown from [16], that this is valid as long as the stress energy tensor for the scalar field is less than the bulk cosmological constant which in turn implies that $v_{h}^{2} / M^{3}$ and $v_{v}^{2} / M^{3}$ are less than unity, where $v_{h}$ and $v_{v}$ are the boundary values of the scalar field. Now using Eqs. (24) and (25), we can determine the conditions of negligible back-reactions in terms of the parameters appearing in the original $F(R)$ theory.

The effect of backreaction, though small, shall also incorporated in Sect. 6.3. We will show that the backreaction modifies all the quantities described via Eqs. (18, 19, 20, 22), though the modification is small in the limit $\kappa v_{h}<1$.

\subsection{Radion potential}

In this section, we consider a fluctuation of branes around the stable separation $\left(r_{c}\right)$. So the inter-brane separation can be considered as a field, and here, for simplicity we assume [17] that this new field depends only on the brane coordinates. The corresponding metric ansatz is,

$d \tilde{s}^{2}=e^{-2 k T(x)|\phi|} \eta_{\mu \nu} d x^{\mu} d x^{\nu}-\tilde{T}^{2}(x)^{2} d \phi^{2}$

From the perspective of four dimensional effective theory, $\tilde{T}(x)$ is known as radion field. Recall that the quantities in tilde are reserved for ST theory. In order to find the radion mass, here we adopt the method proposed by Goldberger and Wise [17]. In the GW mechanism, the same bulk scalar field which stabilize the modulus can also generate the potential for radion field and in the present ST theory (Eq. 15), $\xi(x, \phi)$ can fulfill the purpose.

With the metric in Eq. (26), a Kaluza-Klein reduction for the five dimensional Einstein-Hilbert action reduces to four dimensional effective action as,

$S_{k i n}[\tilde{T}]=\frac{12 M^{3}}{k} \int d^{4} x \partial_{\mu}\left(e^{-k \pi \tilde{T}(x)}\right) \partial^{\mu}\left(e^{-k \pi \tilde{T}(x)}\right)$

As we see that $T(x)$ is not canonical and thus we redefine the field by the following transformation,

$\tilde{T}(x) \longrightarrow \tilde{\Psi}(x)=\sqrt{\frac{24 M^{3}}{k}} e^{-k \pi \tilde{T}(x)}$

In terms of $\tilde{\Psi}$, the kinetic part of radion field becomes

$S_{k i n}[\tilde{\Psi}]=\frac{1}{2} \int d^{4} x\left(\partial_{\mu} \tilde{\Psi}\right)\left(\partial^{\mu} \tilde{\Psi}\right)$

Correspondingly the radion potential is obtained from Eq. (21) by replacing $r_{c}$ by $\tilde{T}(x)$ i.e.

$$
\begin{aligned}
V_{e f f}(\tilde{T})= & k(v+2) A^{2}\left(e^{2 v k \tilde{T}(x) \pi}-1\right) \\
& +k(v-2) B^{2}\left(1-e^{-2 v k \tilde{T}(x) \pi}\right)
\end{aligned}
$$

where $A$ and $B$ are given by,

$$
\begin{aligned}
& A=\frac{1}{\left(1-e^{-2 v k \tilde{T}(x) \pi)}\right.}\left[v_{v} e^{-(2+v) k \tilde{T}(x) \pi}-v_{h} e^{-2 v k \tilde{T}(x) \pi}\right] \\
& B=\frac{1}{\left(1-e^{-2 v k \tilde{T}(x) \pi)}\right.}\left[v_{h}-v_{v} e^{-(2+v) k \tilde{T}(x) \pi}\right]
\end{aligned}
$$

Using the transformation given in Eqs. (27) in (28), we obtain the stable value $(<\tilde{\Psi}>)$ ) and mass squared $\left(\tilde{m}_{\text {rad }}^{2}(S T)\right)$ of the radion field [17] in scalar-tensor theory as

$$
<\tilde{\Psi}>=\sqrt{\frac{24 M^{3}}{k}} e^{-k \pi r_{c}}
$$


with $k \pi r_{c}=\frac{4 k^{2}}{m_{\Phi}^{2}} \ln \left[\frac{v_{h}}{v_{v}}\right]($ see Eq. (22)) and

$\tilde{m}_{r a d}^{2}(S T)=\frac{k^{2} v_{v}^{2}}{3 M^{3}} \epsilon^{2} \frac{e^{-2 k r_{c} \pi}}{\left(1-e^{-2 v k \pi r_{c}}\right)}$

with $\epsilon=m_{\Phi}^{2} / 4 k^{2}$ and $v=\sqrt{4+m_{\Phi}^{2} / k^{2}}$. As mentioned earlier that the solution of gauge hierarchy problem requires $k r_{c} \simeq 12$ for which one can approximate $\left(1-e^{-2 v k \pi r_{c}}\right) \simeq 1$. Under this approximation, the radion mass squared takes the form:

$\tilde{m}_{r a d}^{2}(S T)=\frac{k^{2} v_{v}^{2}}{3 M^{3}} \epsilon^{2} e^{-2 k r_{c} \pi}$

\subsection{Coupling between radion and Standard Model fields}

Being a gravitational degree of freedom, radion field interacts with brane energy-momentum tensor and the couplings of interaction are constrained by four dimensional general covariance. From the five dimensional metric ansatz (see Eq. (26)), it is clear that the induced metric on visible brane is $\left(\frac{\tilde{\Psi}}{f}\right)^{2} \eta_{\mu \nu}$ (where $f=\sqrt{24 M^{3} / k}$ ) and consequently $\tilde{\Psi}(x)$ couples directly with SM fields.

For example, consider the Higgs sector of Standard Model,

$$
\begin{aligned}
S_{\text {Higgs }}= & (1 / 2) \int d^{4} x(\tilde{\Psi} / f)^{4} \\
& {\left[(\tilde{\Psi} / f)^{-2} g^{\mu \nu} \partial_{\mu} h \partial_{\nu} h-\mu_{0}^{2} h^{2}\right] }
\end{aligned}
$$

where $h(x)$ is the Higgs field. In order to get a canonical kinetic term, one needs to redefine $h(x) \longrightarrow H(x)=$ $\frac{<\tilde{\Psi}>}{f} h(x)$. Therefore for $H(x)$, the above action can be written as,

$$
\begin{aligned}
S_{\text {Higgs }}= & (1 / 2) \int d^{4} x\left[\left(\frac{\tilde{\Psi}}{<\tilde{\Psi}>}\right)^{2} g^{\mu \nu} \partial_{\mu} H \partial_{\nu} H\right. \\
& \left.-\left(\frac{\tilde{\Psi}}{<\tilde{\Psi}>}\right)^{4} \mu^{2} H^{2}\right]
\end{aligned}
$$

where $\mu=\mu_{0} \frac{<\tilde{\Psi}>}{f}=\mu_{0} \exp \left[-k \pi r_{c}\right]$. Considering a fluctuation of $\tilde{\Psi}(x)$ about its VEV as $\tilde{\Psi}(x)=<\tilde{\Psi}>+\delta \tilde{\Psi}$, one can obtain (from Eq. (34)) that $\delta \tilde{\Psi}$ couples to $H(x)$ through the trace of the energy-momentum tensor of the Higgs field:

$\mathcal{L}=\frac{\delta \tilde{\Psi}}{\tilde{\Psi}} T_{\mu}^{\mu}(H)$

So, the coupling between radion and Higgs field become, $\lambda_{(H-\delta \tilde{\Psi})}=\frac{\mu^{2}}{<\tilde{\Psi}>}$. Similar consideration holds for any other SM fields. For example for $Z$ boson, the corresponding coupling is $\lambda_{(Z-\delta \tilde{\Psi})}=\frac{m_{Z}^{2}}{<\tilde{\Psi}>}$. Thus the inverse of $<\tilde{\Psi}>$ plays a crucial role in determining the coupling strength between radion and SM fields. In the present case, we obtain (see Eq. (30))
$<\tilde{\Psi}>=\sqrt{\frac{24 M^{3}}{k}} e^{-k \pi r_{c}}$

Hence we finally arrive at,

$\tilde{\lambda}_{(H-\tilde{\Psi})}=\mu^{2} \sqrt{\frac{k}{24 M^{3}}} \exp \left(k \pi r_{c}\right)$

Similarly the coupling between radion and $Z$ boson is,

$\tilde{\lambda}_{(Z-\tilde{\Psi})}=m_{Z}^{2} \sqrt{\frac{k}{24 M^{3}}} \exp \left(k \pi r_{c}\right)$

where $m_{Z}$ is the mass of $Z$ boson.

Now we turn our focus on modulus stabilization as well as on radion mass and coupling for the original $F(R)$ model (Eq. (8)) by using the stabilization condition of the corresponding scalar-tensor theory.

\section{Modulus stabilization, radion mass and coupling in F(R) model}

\subsection{Modulus stabilization}

Recall that the original higher curvature $F(R)$ model is presented by action given in Eq. (8). Solutions of metric $\left(G_{M N}\right)$ for this $F(R)$ model can be extracted from the solutions of corresponding scalar-tensor theory (Eqs. (16) and (18)) with the help of Eq. (9). Thus the line element in $F(R)$ model turns out to be

$d s^{2}=e^{-\frac{\kappa}{\sqrt{3}} \Phi(\phi)}\left[e^{-2 k r_{c}|\phi|} \eta_{\mu \nu} d x^{\mu} d x^{\nu}-r_{c}^{2} d \phi^{2}\right]$

where $\Phi(\phi)=<\Phi>+\xi(\phi)$ and $\xi(\phi)$ is given by Eq. (18). This solution of $G_{M N}$ immediately leads to the separation between hidden $(\phi=0)$ and visible $(\phi=\pi)$ branes along the path of constant $x^{\mu}$ as follows:

$\pi d=r_{c} \int_{0}^{\pi} d \phi e^{-\frac{\kappa}{2 \sqrt{3}} \Phi(\phi)}$

where $d$ is the inter-brane separation in $F(R)$ model. Using the explicit functional form of $\Phi(\phi)$ (Eq. (18)), above equation can be integrated and simplified to the following one,

$k \pi d=k \pi r_{c}-\frac{4 k^{2}}{m_{\Phi}^{2}} \frac{\kappa v_{v}}{2 \sqrt{3}}\left[\frac{v_{h}}{v_{v}}-1\right]$

where the sub-leading terms of $\kappa \xi$ are neglected. $r_{c}$ is the modulus in the corresponding ST theory and it is stabilized which is shown in the previous section (Eq. (22)). So, it can be argued that due to the stabilization of ST theory, the modulus $d$ in $F(R)$ model is also stabilized with a value,

$k \pi d=\frac{4 k^{2}}{m_{\Phi}^{2}}\left[\ln \left(\frac{v_{h}}{v_{v}}\right)-\frac{\kappa v_{v}}{2 \sqrt{3}}\left(\frac{v_{h}}{v_{v}}-1\right)\right]$ 


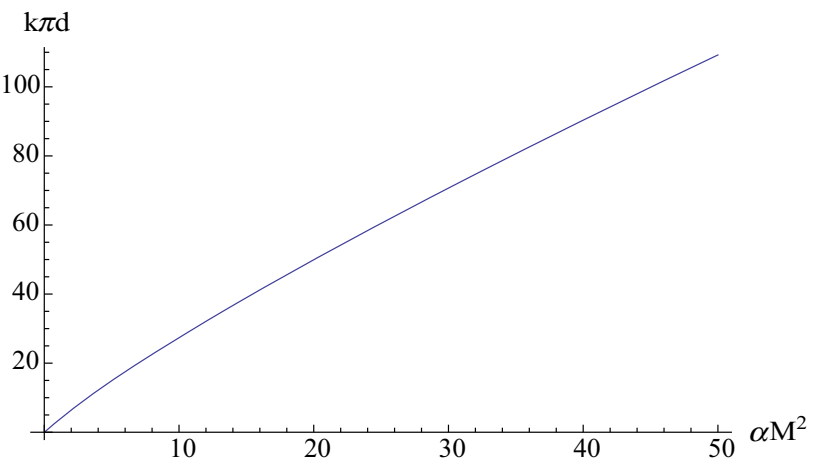

Fig. $1 k \pi d$ vs $|\alpha| M^{2}$

Hence it can be concluded that $F(R)$ model where the only independent field is spacetime metric $\left(G_{M N}\right)$, is a self stabilizing system. From the expression of $m_{\Phi}^{2}$ (Eq. (13)), it is clear that $d$ goes to zero at the limit $\alpha \rightarrow 0$. Moreover for $\alpha>0, m_{\Phi}^{2}$ becomes negative which in turn makes the modulus $d$ negative (see Eq. (39)), an unphysical situation. From the above two statements, it is clear that the self stabilization in $F(R)$ model arises entirely due to the presence of higher curvature term $\alpha R^{2}$ only when $\alpha<0$.

From the above relation (Eq. (39)) and using the expression of $m_{\Phi}^{2}$ (Eq. (13)), we obtain the Fig. 1 between $k \pi d$ and $|\alpha| M^{2}$.

The figure demonstrates that the brane separation $(d)$ increases with the higher curvature parameter $\alpha$. However, using the metric solution given in Eq. (37), one calculates the five dimensional Ricci scalar as follows:

$$
\begin{aligned}
R(\phi)= & -20 k^{2}-\frac{20}{\sqrt{3}} e^{\left[-\frac{\sqrt{3}}{2} \kappa c \phi\right]} k^{2} \kappa c \\
& -5 e^{\left[-2 \frac{\sqrt{3}}{2} \kappa c \phi\right]} k^{2} \kappa^{2} c^{2}
\end{aligned}
$$

where $c$ is an integration constant and $\phi$ is the extra dimensional coordinate. Recall that the boundary values of the curvature (i.e. $R(0)$ and $R(\pi)$, obtained from the Eq. (40)) are related with $v_{h}$ and $v_{v}$ by Eqs. (24) and (25) respectively. Thus one can tune $R(0), R(\pi)$ to fix $v_{h}$ and $v_{v}$ in a desirable value.

\subsection{Radion potential}

A fluctuation of branes around the stable configuration $d$ is now considered. This fluctuation can be taken as a field $(T(x))$ and for simplicity, this new field is assumed to be the function of brane coordinates only. The metric takes the following form,

$$
\begin{gathered}
d s^{2}=e^{-\frac{\kappa}{\sqrt{3}} \Phi(x, \phi)}\left[e^{-2 k T(x)|\phi|} \eta_{\mu \nu} d x^{\mu} d x^{\nu}\right. \\
\left.-T(x)^{2} d \phi^{2}\right]
\end{gathered}
$$

From the angle of four dimensional effective theory, $T(x)$ is known as radion field. Moreover $\Phi(x, \phi)$ is obtained from Eq. (18) by replacing $r_{c}$ to $T(x)$. Plugging the metric solution (Eq. (41)) into the five dimensional $F(R)\left(=R+\alpha R^{2}\right)$ gravitational action $\left(S=\int d^{4} x d \phi \sqrt{G}\left[-\frac{1}{2 \kappa^{2}}\left(R+\alpha R^{2}\right)\right]\right)$ and integrating over $\phi$ yields a kinetic as well as a potential part for the radion field $T(x)$. Kinetic part comes as

$$
\begin{array}{r}
S_{k i n}[T]=\frac{12 M^{3}}{k}\left(1-\frac{40}{\sqrt{3}} \alpha k^{2} \kappa v_{h}\right) \\
\int d^{4} x \partial_{\mu}\left(e^{-k \pi T(x)}\right) \partial^{\mu}\left(e^{-k \pi T(x)}\right)
\end{array}
$$

The signature of higher curvature $R^{2}$ in the above expression comes through the term containing the parameter $\alpha$. It is evident that $T(x)$ is not canonical and in order to make it canonical, we redefine the field as

$T(x) \longrightarrow \Psi(x)=f e^{-k \pi T(x)}$

where $f=\sqrt{\frac{24 M^{3}}{k}}\left[1-\frac{20}{\sqrt{3}} \alpha k^{2} \kappa v_{h}\right]$. For $\alpha \rightarrow 0$, the action contains only the linear term in Ricci scalar and the factor $f$ goes to $\sqrt{24 M^{3} / k}$ which agrees with [17]. With the redefined field, kinetic part of radion field becomes,

$S_{k i n}[\Psi]=\frac{1}{2} \int d^{4} x\left(\partial_{\mu} \Psi\right)\left(\partial^{\mu} \Psi\right)$

Finally the potential part of radion field is given by,

$$
\begin{aligned}
V(\Psi)= & -\frac{20}{\sqrt{3}} \frac{\alpha k^{5}}{M^{6}} \Psi^{4}\left[v_{v}-\left(v_{h}-\frac{\kappa v_{h}^{2}}{2 \sqrt{3}}\right.\right. \\
& \left.\left.+\frac{\kappa v_{h} v_{v}}{2 \sqrt{3}}\right)(\Psi / f)^{\epsilon}\right]^{2}
\end{aligned}
$$

It may be observed that $V(\Psi)$ goes to zero as $\alpha$ tends to zero. This is expected because for $\alpha \rightarrow 0$, the action contains only the Einstein part which does not produce any potential term for the radion field [17]. Thus for five dimensional warped geometric model, the radion potential is generated from the higher order curvature term $\alpha R^{2}$. The potential in Eq. (44) has a vev at

$$
<\Psi>=f\left(\frac{v_{v}}{v_{h}}\right)^{1 / \epsilon}\left[1-\frac{\kappa v_{h}}{2 \sqrt{3}}\left(1-\frac{v_{v}}{v_{h}}\right)\right]^{-1 / \epsilon}
$$

which leads to the interbrane separation as,

$$
\begin{aligned}
k \pi d & =k \pi<T_{F(R)}(x)> \\
& =\frac{4 k^{2}}{m_{\Phi}^{2}}\left[\ln \left(\frac{v_{h}}{v_{v}}\right)-\frac{\kappa v_{h}}{2 \sqrt{3}}\left(1-\frac{v_{v}}{v_{h}}\right)\right]
\end{aligned}
$$

in the leading order of $\kappa \xi$. This above equation exactly resembles with the Eq. (39) which again indicates that the five dimensional warped spacetime we considered, is self stabilized by higher curvature gravitational theory. We emphasize that due to the presence of conformal factor connecting the 
two theories, the value of $k \pi d$ (in $F(R)$ model) is less than $k \pi r_{c}$ (in ST model). However we find that the stabilization of modulus remain intact in both the models. Finally the squared mass of radion field is as follows,

$$
\begin{aligned}
m_{\text {rad }}^{2}(F(R))= & -\frac{20}{\sqrt{3}} \frac{\alpha k^{4}}{M^{6}} \epsilon^{2} e^{-2 k d \pi} v_{h}^{2} v_{v}^{2} \\
& {\left[1-\frac{40}{\sqrt{3}} \alpha k^{2} \kappa v_{h}\right]\left[\frac{v_{h}}{v_{v}}-1\right]^{2} }
\end{aligned}
$$

It is noticed that the mass of radion field is enhanced by the higher curvature terms in five dimensional gravitational action.

\subsection{Radion potential with backreaction}

It may be mentioned that the stabilized interbrane separation obtained in Eq. (46) is based on the conditions that the bulk scalar potential is retained up to quadratic term (see Eq. (15)) and the backreaction of the bulk scalar field is neglected on five dimensional spacetime. Both these conditions are followed from the assumption that $\kappa v_{h}<1$. Relaxation of this assumption is crucial to check the status of the stabilization condition in the presence of higher order self interaction terms in the bulk scalar potential. Here we examine whether the radion potential admits any stability when $V(\Phi)$ is retained up to cubic term in $\Phi$. In this scenario, the five dimensional action in ST theory turns out to be,

$$
\begin{aligned}
S= & \int d^{4} x d \phi \sqrt{\tilde{G}}\left[-\frac{\tilde{R}}{2 \kappa^{2}}+\frac{1}{2} \tilde{G}^{M N} \partial_{M} \xi \partial_{N} \xi\right. \\
& \left.-(1 / 2) m_{\Phi}^{2} \xi^{2}-\frac{g}{3} \xi^{3}-\Lambda_{e f f}\right]
\end{aligned}
$$

where $g$ is the self cubic coupling of $\Phi(\phi)$ and can be easily determined from the form of $V(\Phi)$ presented in Eq. (11) as,

$g=\frac{\sqrt{3} \kappa}{16 \alpha} \frac{\left[2 \sqrt{9-40 \kappa^{2} \alpha \Lambda}+3\right]}{\left[\sqrt{9-40 \kappa^{2} \alpha \Lambda}-2\right]^{\frac{2}{3}}}$

Considering the metric ansatz as,

$d \tilde{s}^{2}=e^{-2 A(\phi)} \eta_{\mu \nu} d x^{\mu} d x^{\nu}-r_{c}^{2} d \phi^{2}$

the gravitational as well as the scalar field equations of motion take the following form,

$$
\begin{gathered}
\frac{4}{r_{c}^{2}} A^{\prime 2}(\phi)-\frac{1}{r_{c}^{2}} A^{\prime \prime}(\phi)=-\left(2 \kappa^{2} / 3\right)\left(\frac{1}{2} m_{\Phi}^{2} \xi^{2}+\frac{g}{3} \xi^{3}\right) \\
\frac{1}{r_{c}^{2}} A^{\prime 2}(\phi)=\frac{\kappa^{2}}{12 r_{c}^{2}} \xi^{\prime 2}-\left(\kappa^{2} / 6\right)\left(\frac{1}{2} m_{\Phi}^{2} \xi^{2}+\frac{g}{3} \xi^{3}\right) \\
\frac{1}{r_{c}^{2}} \xi^{\prime \prime}(\phi)=f r a c 4 r_{c}^{2} A^{\prime} \xi^{\prime}+m_{\Phi}^{2} \xi(\phi)+g \xi^{2}(\phi)
\end{gathered}
$$

To determine the solutions of the above differential equations, we apply the iterative method by considering the form of metric determined in Eq. (16) as the zeroth order solution. In the leading order correction of $\kappa v_{h}, \xi(\phi)$ and $A(\phi)$ turn out to be

$$
\begin{aligned}
\xi(\phi)= & {\left[A e^{(2+v) k r_{c} \phi}+B e^{(2-v) k r_{c} \phi}\right] } \\
& +\frac{\kappa v_{h}}{16 \sqrt{3} \alpha k^{2}} \frac{\left[2 \sqrt{9-40 \kappa^{2} \alpha \Lambda}+3\right]}{\left[\sqrt{9-40 \kappa^{2} \alpha \Lambda}-2\right]^{\frac{2}{3}}} \\
& *\left[\frac{\exp \left[2(2+v) k r_{c} \phi-4 v k r_{c} \pi\right]}{m_{\Phi}^{2} r_{c}^{2}+8 k r_{c}(2+v)-4(2+v)^{2}}\right. \\
& \left.+\frac{2 v_{v}}{v_{h}} \frac{\exp \left[4 k r_{c} \phi-(2+v) k r_{c} \pi\right]}{m_{\Phi}^{2} r_{c}^{2}+16 k r_{c}-16}\right]
\end{aligned}
$$

and

$$
\begin{aligned}
A(\phi)= & k r_{c} \phi+\frac{\kappa^{2} v_{h}^{2}}{12}\left[e^{-4 v k r_{c} \pi} e^{-2(2+v) k r_{c} \phi}\right. \\
& \left.+e^{2(2-v) k r_{c} \phi}\left(1+\frac{v_{v}}{v_{h}} e^{-(2+v) k r_{c} \pi}\right)\right]
\end{aligned}
$$

Thus due to the inclusion of the bulk scalar field backreaction, the warp factor gets modified and the correction term is proportional to $\kappa^{2} v_{h}^{2}$ which is indeed small for $\kappa v_{h}<1$. However if one includes these corrections in $\xi(\phi)$ and $A(\phi)$, one can extract the modified solution in $\mathrm{F}(\mathrm{R})$ model by a conformal transformation as indicated earlier:

$$
\begin{aligned}
d s^{2}= & \exp \left[-\frac{\kappa}{\sqrt{3}}(<\Phi>+\xi(\phi))\right] * \\
& {\left[e^{-2 A(\phi)} \eta_{\mu \nu} d x^{\mu} d x^{\nu}-r_{c}^{2} d \phi^{2}\right] }
\end{aligned}
$$

where $d s^{2}$ is the line element in $F(R)$ model and $\xi(\phi), A(\phi)$ are given in Eqs. (54) and (55) respectively. Plugging back the above solution of metric (in Eq. (56)) into the five dimensional $F(R)$ action and integrating over $\phi$, the radion potential is obtained as

$$
\begin{aligned}
V(\Psi)= & -\frac{20}{\sqrt{3}} \frac{\alpha k^{5}}{M^{6}} \Psi^{4}\left[v_{v}-\left(v_{h}-\frac{\kappa v_{h}^{2}}{2 \sqrt{3}}+\frac{\kappa v_{h} v_{v}}{2 \sqrt{3}}\right.\right. \\
& \left.\left.+\frac{\kappa^{2} v_{h}^{3}}{64 \sqrt{3}}-\frac{\kappa^{2} v_{h}^{2} v_{v}}{616 \sqrt{3}}+\frac{\kappa^{2} v_{v}^{2} v_{h}}{16 \sqrt{3}}\right)(\Psi / f)^{\epsilon}\right]^{2}
\end{aligned}
$$

The above potential has a stable minimum at,

$$
\begin{aligned}
<\Psi>= & f\left(\frac{v_{v}}{v_{h}}\right)^{1 / \epsilon}\left[1-\frac{\kappa v_{h}}{2 \sqrt{3}}\left(1-\frac{v_{v}}{v_{h}}\right)+\frac{\kappa^{2} v_{h}^{2}}{64 \sqrt{3}}\right. \\
& \left.-\frac{\kappa^{2} v_{h} v_{v}}{16 \sqrt{3}}+\frac{\kappa^{2} v_{v}^{2}}{16 \sqrt{3}}\right]^{-1 / \epsilon}
\end{aligned}
$$

where $\epsilon=m_{\Phi}^{2} / 4 k^{2}$ and $m_{\Phi}^{2}$ is given in Eq. (13). Above expression of $\langle\Psi\rangle$ leads to the stabilized interbrane separation as:

$k \pi d=k \pi<T_{F(R)}(x)>$ 


$$
\begin{aligned}
= & \frac{4 k^{2}}{m_{\Phi}^{2}}\left[\ln \left(\frac{v_{h}}{v_{v}}\right)-\frac{\kappa v_{h}}{2 \sqrt{3}}\left(1-\frac{v_{v}}{v_{h}}\right)\right. \\
& \left.+\frac{\kappa^{2} v_{h}^{2}}{64 \sqrt{3}}\left(1-4 \frac{v_{v}}{v_{h}}+4 \frac{v_{v}^{2}}{v_{h}^{2}}\right)\right]
\end{aligned}
$$

Comparing Eqs. (46) and (59), it can be seen that the vev of the radion field and hence the stable modulus is shifted due to the presence of higher order self interaction terms in the bulk scalar potential or the inclusion of scalar field backreaction. However this shift is indeed small in the limit $\kappa v_{h}<1$.

\subsection{Coupling between radion and Standard Model fields}

$\mathrm{s}$ The radion field arises as a scalar degree of freedom on the TeV brane and has interactions with the Standard Model (SM) fields. From the five dimensional metric (Eq. (41)), it is clear that the induced metric on visible brane is $\left(\frac{\Psi}{f}\right)^{2} e^{-\kappa v_{v} \frac{1}{\sqrt{3}}} \eta_{\mu \nu}$ (where $\left.f=\sqrt{\frac{24 M^{3}}{k}}\left[1-\frac{20}{\sqrt{3}} \alpha k^{2} \kappa v_{h}\right]\right)$ and consequently $\Psi(x)$ couples directly with SM fields.

For example, consider the Higgs sector of Standard Model,

$$
\begin{aligned}
S_{\text {Higgs }}= & (1 / 2) \int d^{4} x(\Psi / f)^{4} e^{-\kappa v_{v} \frac{2}{\sqrt{3}}} \\
& {\left[(\Psi / f)^{-2} e^{\kappa v_{v} \frac{1}{\sqrt{3}}} \eta^{\mu \nu} \partial_{\mu} h \partial_{\nu} h-\mu_{0}^{2} h^{2}\right] }
\end{aligned}
$$

where $h(x)$ is the Higgs field. In order to get a canonical kinetic term, one needs to redefine $h(x) \longrightarrow H(x)=$ $\frac{<\Psi>}{f} h(x)$. Therefore for $H(x)$, the above action can be written as,

$$
\begin{aligned}
S_{\text {Higgs }}= & (1 / 2) \int d^{4} x\left[\left(\frac{\Psi}{<\Psi>}\right)^{2} \eta^{\mu \nu} \partial_{\mu} H \partial_{\nu} H\right. \\
& \left.-\left(\frac{\Psi}{<\Psi>}\right)^{4} \mu^{2} H^{2}\right]
\end{aligned}
$$

where $\mu=\mu_{0} \frac{<\Psi>}{f} e^{-\kappa v_{v} \frac{1}{\sqrt{3}}}$. Considering a fluctuation of $\Psi(x)$ about its vev as $\Psi(x)=<\Psi>+\delta \Psi$, one can obtain (from Eq. (61)) that $\delta \Psi$ couples to $H(x)$ through the trace of the energy-momentum tensor of the Higgs field:

$\mathcal{L}=\frac{\delta \Psi}{<\Psi>} T_{\mu}^{\mu}(H)$

So, the coupling between radion and Higgs field become, $\lambda_{(H-\delta \Psi)}=\frac{\mu^{2}}{<\Psi>}$. Similar consideration holds for any other $\mathrm{SM}$ fields. For example for $Z$ boson, the corresponding coupling is $\lambda_{(Z-\delta \Psi)}=\frac{m_{Z}^{2}}{\langle\Psi\rangle}$. Thus the inverse of $\langle\Psi\rangle$ plays a crucial role in determining the coupling strength between radion and SM fields. In the present case, we obtain

$<\Psi>=f e^{-k \pi d}$

Hence finally we arrive at,

$$
\begin{aligned}
\lambda_{(H-\delta \Psi)}= & \mu^{2} \sqrt{\frac{k}{24 M^{3}}} e^{k \pi d} \\
& *\left[1+\frac{20}{\sqrt{3}} \alpha k^{2} \kappa v_{h}\right]
\end{aligned}
$$

and

$$
\begin{aligned}
\lambda_{(Z-\delta \Psi)}= & m_{Z}^{2} \sqrt{\frac{k}{24 M^{3}}} e^{k \pi d} \\
& *\left[1+\frac{20}{\sqrt{3}} \alpha k^{2} \kappa v_{h}\right]
\end{aligned}
$$

in the leading order of $\kappa$.

The coupling between radion and fermion field is similarly given by,

$\lambda_{(\text {fermion }-\delta \Psi)}=\sqrt{\frac{k}{24 M^{3}}} e^{k \pi d}\left[1+\frac{20}{\sqrt{3}} \alpha k^{2} \kappa v_{h}\right]$

If the fermion fields are allowed to propagate in the bulk, then the coupling with radion changes from that given in (Eq. (64)). This can be determined by Kaluza-Klein (KK) mode expansion of the bulk fermion [47] in a bulk governed by $F(R)$ gravity. Here we focus on the zeroth order KK mode and the solution of the zeroth order KK mode wave function in the bulk takes the form:

$\chi_{L}(\phi)=\left[\sqrt{9-40 \kappa^{2} \alpha \Lambda}-2\right]^{-\frac{1}{3}} \sqrt{\frac{(k+2 m) d}{e^{(k+2 m) \pi d}-1}} e^{m d \phi}$

where $\chi_{L}(\phi)$ is the left handed fermion wave function and $m$ is bulk fermionic mass. Recall that $d$ is the interbrane separation and $<\Phi>$ is given in Eq. (12). Similarly for the right handed mode $\left(\chi_{R}(\phi)\right)$,

$$
\chi_{R}(\phi)=\left[\sqrt{9-40 \kappa^{2} \alpha \Lambda}-2\right]^{-\frac{1}{3}} \sqrt{\frac{(k-2 m) d}{e^{(k-2 m) \pi d}-1}} e^{-m d \phi}
$$

Using these above solutions, we determine the coupling of radion with zeroth order fermionic KK mode, which yields,

$$
\begin{gathered}
\lambda_{L}=\sqrt{\frac{k}{24 M^{3}} e^{k \pi d}\left[1+\frac{20}{\sqrt{3}} \alpha k^{2} \kappa v_{h}\right]} \\
{\left[\sqrt{9-40 \kappa^{2} \alpha \Lambda}-2\right]^{-\frac{2}{3}}(k+2 m) d} \\
\left(\frac{e^{(k+2 m) \pi d}}{e^{(k+2 m) \pi d}-1}\right)
\end{gathered}
$$

for left handed chiral mode and,

$$
\begin{gathered}
\lambda_{R}=\sqrt{\frac{k}{24 M^{3}} e^{k \pi d}\left[1+\frac{20}{\sqrt{3}} \alpha k^{2} \kappa v_{h}\right]} \\
{\left[\sqrt{9-40 \kappa^{2} \alpha \Lambda}-2\right]^{-\frac{2}{3}}(k-2 m) d} \\
\left(\frac{e^{(k-2 m) \pi d}}{e^{(k-2 m) \pi d}-1}\right)
\end{gathered}
$$


for right handed mode.

The form of $v_{h}$ and $v_{v}$ in terms of five dimensional Ricci scalar can be extracted from Eqs. (24) and (25). It is evident that the coupling $\lambda_{L}, \lambda_{R}$ is modified by the factor $(k \pm 2 m) d$, in comparison to the coupling given in Eq. (64) for the fermion fields confined on the $\mathrm{TeV}$ brane.

From above analysis we note that the coupling between radion and SM fields is suppressed due to the presence of higher curvature parameter $\alpha$ which in turn modifies the phenomenology on visible 3-brane.

Before concluding, we mention about a recent work [46], where a higher curvature gravity model with $R^{4}$ terms in the action is considered. The corresponding conformally transformed scalar action includes a quartic term which resembles closely to the scalar action considered in reference $[11,13]$. It has been shown that with such a specific choice of the scalar action one can estimate the exact modification of the warp factor due to the effects of the back-reaction of the scalar field on the background geometry and thus it enables us to address the role of back-reaction on the stabilization issue and also on various parameters of the low-energy effective action. However in such a model, the back-reacted geometry can be exactly determined if the scalar mass and the quartic term in the potential are inter-related and in the limit of the quartic term going to zero, the mass term also goes to zero. Therefore there is no smooth limit which takes this model to that considered by the GW where only quadratic mass term was present. The work reported in this article however has a different goal from that of [46]. Here we show that in the leading order quadratic curvature correction to Einstein action in the bulk we find a dual scalar tensor theory which under certain approximation is similar to the original GW scalar action which has a quadratic mass term only. We therefore explore and re-examine the originally proposed Goldberger-Wise modulus stabilization condition in the light of higher curvature gravity models where such a stabilizing field appears naturally from higher curvature degrees of freedom with a minimal curvature extension.

\section{Conclusion}

In this work, we consider a five dimensional AdS, compactified warped geometry model with two 3-branes embedded within the spacetime. Due to large curvature ( Planck scale) in the bulk, the spacetime is assumed to be governed by a higher curvature gravity model , $F(R)=R+\alpha R^{2}$. In this scenario, we address how the modulus stabilization and radion phenomenology are affected by higher curvature term. The findings and implications of our results are as follows:

- The model comes as a self stabilizing system due to the presence of higher curvature term $\alpha R^{2}$. This is in sharp contrast to a model with only Einstein term in the bulk where the modulus can not be stabilized without incorporating any external degrees of freedom such as a scalar field. However for the higher curvature gravity model, this additional degree of freedom originates naturally from the higher curvature term and plays the role of a stabilizing field. It may also be noted that for $\alpha \rightarrow 0$, the stabilization condition (Eq. (39)) leads to zero brane separation.

- We scan the parametric space of $\alpha$ for which the modulus is going to be stabilized. Our result reveals that for $\alpha>0$, the interbrane separation becomes negative which is an unphysical situation. Thus the braneworld we have considered is stabilized only when $\alpha<0$. This puts constraints on the $F(R)$ model itself. Moreover the distance between the branes increases with the value of the parameter $\alpha$ which is evident from Fig. 1. Thus the results obtained in this work clearly bring out the correlation between a geometrically stable warped solution resulting from negative bulk curvature and the stability of the higher curvature $F(R)$ model free from ghosts.

- Quadratic term in curvature also generates the radion potential with a stable minimum. We find the radion mass as well as radion coupling with SM fields. The expressions of mass (Eq. (47)) and coupling (Eq. (62) and Eq. (63)) clearly indicate that the radion mass is enhanced while the coupling is suppressed in comparison to the scenario where only Einstein gravity resides in the bulk [17]. Thus the cross section between radion and SM fields is overall suppressed due to the presence of higher order curvature terms in five dimensional gravitational action leading to a possible explanation of the invisibility of the radion field in the present experimental resolution.

- The effect of backreaction on the radion potential and its minima are studied. It is shown that the corrections due to the backreaction is indeed small in the limit $\kappa v_{h}<1$. The possible correction terms for the backreaction are determined.

Open Access This article is distributed under the terms of the Creative Commons Attribution 4.0 International License (http://creativecomm ons.org/licenses/by/4.0/), which permits unrestricted use, distribution, and reproduction in any medium, provided you give appropriate credit to the original author(s) and the source, provide a link to the Creative Commons license, and indicate if changes were made. Funded by $\mathrm{SCOAP}^{3}$.

\section{References}

1. N. Arkani-Hamed, S. Dimopoulos, G. Dvali, Phys. Lett. B 429, $263(1998)$ 
2. N. Arkani-Hamed, S. Dimopoulos, G. Dvali, Phys. Rev. D 59, 086004 (1999)

3. I. Antoniadis, N. Arkani-Hamed, S. Dimopoulos, G. Dvali, Phys. Lett. B 436, 257 (1998)

4. P. Horava, E. Witten, Nucl. Phys. B 475, 94 (1996)

5. P. Horava, E. Witten, Nucl. Phys. B 460, 506 (1996)

6. L. Randall, R. Sundrum, Phys. Rev. Lett. 83, 3370 (1999)

7. N. Kaloper, Phys. Rev. D 60, 123506 (1999)

8. T. Nihei, Phys. Lett. B 465, 81 (1999)

9. H.B. Kim, H.D. Kim, Phys. Rev. D 61, 064003 (2000)

10. A.G. Cohen, D.B. Kaplan, Phys. Lett. B 470, 52 (1999)

11. C.P. Burgess, L.E. Ibanez, Phys. Lett. B 447, 257 (1999)

12. A. Chodos, E. Poppitz, Phys. Lett. B 471, 119 (1999)

13. T. Gherghetta, M. Shaposhnikov, Phys. Rev. Lett. 85, 240 (2000)

14. M.B. Green, J.H. Schwarz, E. Written, “Superstring Theory”, Vol.I and Vol.II (Cambridge University Press, Cambridge, 1987)

15. J. Polchinski, String theory (Cambridge University Press, Cambridge, 1998)

16. W.D. Goldberger, M.B. Wise, Phys. Rev. Lett. 83, 4922 (1999)

17. W.D. Goldberger, M.B. Wise, Phys. Lett B 475, 275-279 (2000)

18. C. Csaki, M.L. Graesser, D. Graham, Kribs. Phys. Rev. D. 63, $065002(2001)$

19. J. Lesgourgues, L. Sorbo, Goldberger-Wise variations: stabilizing brane models with a bulk scalar. Phys. Rev. D 69, 084010 (2004)

20. O. DeWolfe, D.Z. Freedman, S.S. Gubser, A. Karch, Phys. Rev. D. 62, $046008(2000)$

21. H. Davoudiasl, J.L. Hewett, T.G. Rizzo, Phys. Rev. Lett. 84, 2080 (2000)

22. T.G. Rizzo, Int. J. Mod. Phys A15, 2405-2414 (2000)

23. Y. Tang, JHEP 1208, 078 (2012)

24. H. Davoudiasl, J.L. Hewett, T.G. Rizzo, JHEP 0304, 001 (2003)

25. M.T. Arun, D. Choudhury, A. Das, S. Sengupta, Phys. Lett. B 746, 266-275 (2015)

26. P. Figueras, T. Wiseman, Gravity and large black holes in RandallSundrum II Braneworlds. PRL 107, 081101 (2011)

27. N. Dadhich, R. Maartens, P. Papadopoulos, V. Rezania : Black Holes on the Brane, Phys. Lett. B487 (2000)

28. D.C. Dai, D. Stojkovic, Analytic solution for a static black hole in RSII model. Phys. Lett. B 704, 354-359 (2011)

29. ATLAS Collaboration, Phys. Lett. B710, 538-556 (2012)

30. ATLAS Collaboration, G. Aad et al, Phys. Rev. D. 90, 052005 (2014)
31. T.P. Sotiriou, V. Faraoni, $f(R)$ theories of gravity. Rev. Mod. Phys. 82, 451497 (2010). arXiv:0805.1726 [gr-qc]

32. A. De Felice, $\mathrm{S}$. Tsujikawa, $\mathrm{f}(\mathrm{R})$ theories. Living Rev. Relat. 13, 3 (2010). arXiv: 1002.4928 [gr-qc]

33. A.Paliathanasis, $f(R)$-gravity from Killing Tensors, Class. Quant. Grav. 33no. 7, 075012 (2016), arXiv:1512.03239 [gr-qc]

34. S.Nojiri, S. D. Odintsov, Phys. Lett. B 631 (2005) 1.arxiv:hepth/0508049

35. S. Nojiri, S.D. Odintsov, O.G. Gorbunova, J. Phys. A 39, 6627 (2006). arxiv:hep-th/0510183

36. G. Cognola, E. Elizalde, S. Nojiri, S.D. Odintsov, S. Zerbini, Phys. Rev. D 73, 084007 (2006)

37. J.E.Kim, B. Kyae, H.M. Lee, Phys.Rev D62, 045013 (2000), arxiv: hepph/9912344

38. J.E. Kim, B. Kyae, H.M. Lee, Nucl. Phys. B582, 296 (2000), Erratum. Nucl. Phys. B591, 587 (2000). hep-th/0004005

39. S. Choudhury, S. SenGupta, JHEP 1302, 136 (2013)

40. J.D. Barrow, S. Cotsakis, Inflation and the conformal structure of higher order gravity theories. Phys. Lett. B 214, 515-518 (1988)

41. S. Capozziello, R. de Ritis, A. A. Marino, Some aspects of the cosmological conformal equivalence between Jordan frame and Einstein frame, Class. Quant. Grav. 14, 32433258 (1997), arXiv: gr-qc/9612053 [gr-qc]

42. S. Bahamonde, S. D. Odintsov, V. K. Oikonomou, M. Wright, Correspondence of $\mathrm{F}(\mathrm{R})$ Gravity Singularities in Jordan and Einstein Frames, arXiv: 1603.05113 [gr-qc]

43. R. Catena, M. Pietroni, L. Scarabello, Einstein and Jordan reconciled: a frame-invariant approach to scalar-tensor cosmology, Phys. Rev. D76, 084039 (2007), arXiv: astro-ph/0604492 [astro-ph]

44. S. SenGupta, S. Chakraborty, "Solving higher curvature gravity theories", Eur. Phys. J. C76, no.10, 552 (2016) arXiv: 1604.05301

45. S. Anand, D. Choudhury, Anjan A. Sen, S. SenGupta, "A Geometric Approach to Modulus Stabilization" Phys. Rev. D92, no.2, 026008 (2015), arXiv: 1411.5120

46. S. Chakraborty, S. SenGupta, arXiv: 1701.01032

47. Y. Grossman, M. Neubert, Phys. Lett. B 474, 361-371 (2000) 